\title{
Meibomian gland dysfunction, dropout and distress: emerging therapies
}

\author{
Ali Hassan ${ }^{1,2} \cdot$ Shafi Balal $\mathbb{C}^{1,2} \cdot$ Sajjad Ahmad ${ }^{1,2}$ \\ Received: 22 February 2020 / Revised: 26 February 2020 / Accepted: 28 February 2020 / Published online: 8 April 2020 \\ (c) The Author(s), under exclusive licence to The Royal College of Ophthalmologists 2020
}

Our understanding of the multifactorial origin and complexity of meibomian gland disease (MGD) is evolving rapidly and new treatments are emerging. A recent randomised trial comparing lifitegrast ophthalmic solution to thermal pulsation for the treatment of inflammatory MGD [1] highlights advances made in treating this frequently disabling condition.

In this editorial, we will describe these emerging therapies and any evidence for their use. Disease of the meibomian glands is commonly encountered in clinical practice [2]. Mild disease can often be over-diagnosed and over-treated and severe disease, particularly if associated with ocular surface inflammatory diseases, can be overlooked or undertreated [3]. The overall impact of MGD is a loss of the normal lipid layer of the tear film that can result in an evaporative form of dry eye disease (DED). In severe disease, there is permanent gland atrophy, scarring and erosion of the posterior lid margin, altered microbial flora of the ocular surface and chronic inflammation [4].

MGD is not a single entity but multifactorial in origin. Causes include congenital absence of meibomian glands such as in ectodermal dysplasia; abnormal blinking (reduced rate and amplitude) seen in conditions like dementia and Parkinson's disease and compromising lipid outflow; obstruction of the meibomian terminal ductules such as in Stevens Johnson syndrome due to keratinization of the lid margin; structural damage to the glands caused by cicatricial diseases; inflammation of the glands such as in rosacea; and direct disruption of the lipid layer caused by topical medications [5].

Ali Hassan

alihassan@nhs.net

1 Moorfields Eye Hospital NHS Foundation Trust, 162 City Road, London EC1V 2PD, UK

2 Institute of Ophthalmology, University College London, 11-43 Bath Street, London EC1V 9EL, UK
Conventional management of MGD involves warm eyelid compresses (increasingly with microwaveable bead-filled bags) and systemic antibiotics (oral doxycycline or erythromycin) [6]. However, there are now several new and adjunctive treatment approaches, either as devices or topical/ systemic therapies:

(1) Lipiflow is an FDA approved thermal device, which applies $42.5^{\circ} \mathrm{C}$ heat to the palpebral eyelid surfaces with concurrent proximal to distal peristaltic pressure over the meibomian glands. A non-randomised interventional trial of fifty patients found a single Lipiflow treatment to be as effective as a 3-month treatment of twice daily conventional lid compresses [7], and a randomised control trial (RCT) of twentyeight patients found it to be possibly more effective than 3-months of oral doxycycline [8]. A NICE evidence review in 2015 prior to these two trials determined that there was insufficient evidence to evaluate Lipiflow for NICE guidance. Lipiflow has significant set-up and on-going consumable costs.

(2) Intense pulsed light (IPL) therapy is a non-invasive treatment using high-energy flashes of light between $400-1200 \mathrm{~nm}$ lasting several milliseconds. This is converted into heat resulting in selective tissue thermolysis [9]. The mechanism of action of IPL in MGD is putatively related to thermocoagulation of vascular telangiectasia, antimicrobial effects and a direct effect on abnormal lipids. Data from five double masked RCTs showed that IPL either alone [10-12] or combined with gland expression [13] results in improved symptoms and tear film stability with one study also reporting a reduction in the levels of inflammatory markers in tear fluid [14]. IPL for MGD must be performed with scleral shield protection of the globe as intraocular damage has been reported [15].

(3) Azithromycin is a macrolide antibiotic with antiinflammatory and anti-microbial effects. It stimulates 
the accumulation of intracellular phospholipids and lysosomes which are important in the maturation of meibocytes [16]. The use of oral [17], topical [18] and combined [19] azithromycin for MGD is supported by clinical trial evidence, however, optimal dosage, frequency and duration of topical and/or systemic azithromycin for MGD however remains to be determined.

(4) Oral omega-3 essential fatty acids are anti-inflammatory and can qualitatively improve meibum [20]. A systematic review appraising omega-3 and omega-6 supplementation for DED found the best results were obtained in MGD patients [21]. However a recent large DREAM ${ }^{\circledast}$ multicenter RCT showed no difference compared to an olive oil control. It must, however, be noted that this latter study, however, had fewer inclusion criteria and was not MGD specific [22].

(5) Topical Ciclosporin A (CsA) inhibits T-cell proliferation and activation and is licensed and NICE approved for the treatment of dry eye disease. Due to its biological effects, CsA reduces ocular surface inflammation that has been implicated in the pathogenesis and seen in the sequelae of MGD [4]. CsA has been shown to improve lipid layer parameters in at least eight clinical trials for DED to date [23]. One retrospective case review study of 53 patients specifically with MGD showed improvement in symptom scores and some signs of MGD including tear break up time [24].

(6) Lifitegrast is an FDA approved topical immunomodulatory medication for dry eye disease. It blocks T-cell binding to a number of integrin's thereby downregulating inflammation [25]. The OPUS-2 RCT was a large multicentre study comparing lifitegrast versus placebo in 718 subjects with DED that showed symptom improvement. However, patients undergoing topical or systemic treatment for MGD were excluded from this large study. A recent RCT in patients specifically with MGD (evident gland blockage) compared lifitegrast to Lipiflow treatment in only 50 patients. This showed lifitegrast superiority for the treatment of MGD [1]. Lifitegrast is currently in the NICE pipeline for evaluation.

(7) Demodex is the most common parasite found on human skin, and the mite and its excreta can directly block gland orifices resulting in MGD [26]. Lash sampling and microscopy are required for confirmatory diagnosis however cylindrical debris at the lash base, misdirected and brittle lashes in MGD not responsive to conventional therapies should raise suspicion. Diluted tea tree oil treatment of the lids has been shown to reduce mite counts however this can cause contact dermatitis in some patients. Other agents such as pilocarpine gel and povidone iodine are also being investigated for eyelid Demodex eradication [27].

In addition to the aforementioned, several other treatment options for MGD are in the pipeline, including the P2Y2 receptor agonist Diquafosol [28] and Manuka honey eye preparations [29] that have been shown to improve MGD however further studies are needed to establish effectiveness.

The therapeutic landscape in MGD is rapidly evloving with the last ten years seeing a sevenfold increase in the number of studies published annually, and a ever increasing number of patents being filed for MGD therapies. As we begin to understand MGD better as a result of newer imaging modalities and assays for inflammation, we now have an increasing array of novel therapies to offer our severely symptomatic patients with obvious MGD.

\section{Compliance with ethical standards}

Conflict of interest The authors declare that they have no conflict of interest.

Publisher's note Springer Nature remains neutral with regard to jurisdictional claims in published maps and institutional affiliations.

\section{References}

1. Tauber J. A 6-week, prospective, randomized, single-masked study of lifitegrast ophthalmic solution $5 \%$ versus thermal pulsation procedure for treatment of inflammatory meibomian gland dysfunction. Cornea. 2020;39(Apr):403-7.

2. Schaumberg DA, Nichols JJ, Papas EB, Tong L, Uchino M, Nichols KK. The international workshop on meibomian gland dysfunction: report of the subcommittee on the epidemiology of, and associated risk factors for, MGD. Investig Ophthalmol Vis Sci. 2011;52:1994-2005.

3. Shimazaki J, Goto E, Ono M, Shimmura S, Tsubota K. Meibomian gland dysfunction in patients with Sjögren syndrome. Ophthalmology. 1998;105:1485-8.

4. Baudouin C, Messmer EM, Aragona P, Geerling G, Akova YA, Benítez-del-Castillo J, et al. Revisiting the vicious circle of dry eye disease: a focus on the pathophysiology of meibomian gland dysfunction. Br J Ophthalmol. 2016;100:300-6.

5. Bron AJ, de Paiva CS, Chauhan SK, Bonini S, Gabison EE, Jain S, et al. Tfos dews ii pathophysiology report. Ocul Surf. 2017;15: 438-510.

6. Nichols KK, Foulks GN, Bron AJ, Glasgow BJ, Dogru M, Tsubota $\mathrm{K}$, et al. The international workshop on meibomian gland dysfunction: executive summary. Investig Ophthalmol Vis Sci. 2011;52:1922-9.

7. Zhao Y, Veerappan A, Yeo S, Rooney DM, Acharya RU, Tan JH. et al. Clinical trial of thermal pulsation (LipiFlow) in meibomian gland dysfunction with preteatment meibography. Eye Contact Lens. 2016;42:339

8. Hagen KB, Bedi R, Blackie CA, Christenson-Akagi KJ. Comparison of a single-dose vectored thermal pulsation procedure with a 3-month course of daily oral doxycycline for moderate-to-severe meibomian gland dysfunction. Clin Ophthalmol (Auckl, NZ). 2018;12:161. 
9. Raulin C, Greve B, Grema H. IPL technology: a review. Lasers Surg Med. 2003;32:78-87.

10. Craig JP, Chen YH, Turnbull PR. Prospective trial of intense pulsed light for the treatment of meibomian gland dysfunction. Invest Ophthalmol Vis Sci. 2015;56:1965-70.

11. Arita R, Fukuoka S, Morishige N. Therapeutic efficacy of intense pulsed light in patients with refractory meibomian gland dysfunction. Ocul Surf. 2019;17:104-10.

12. Rong B, Tang Y, Tu P, et al. Intense pulsed light applied directly on eyelids combined with meibomian gland expression to treat meibomian gland dysfunction. Photomed Laser Surg. 2018;36:326-32.

13. Rong B, Tang Y, Liu R, Tu P, Qiao J, Song W, et al. Long-term effects of intense pulsed light combined with meibomian gland expression in the treatment of meibomian gland dysfunction. Photomed Laser Surg. 2018;36:562-7.

14. Liu R, Rong B, Tu P, Tang Y, Song W, Toyos R, et al. Analysis of cytokine levels in tears and clinical correlations after intense pulsed light treating meibomian gland dysfunction. Am J Ophthalmol. 2017;183:81-90.

15. Ho T, Teo L. Retinal toxicity from intense pulsed light therapy: a case report. EC Ophthalmol. 2020;11:1-4.

16. Liu Y, Kam WR, Ding J, Sullivan DA. One man's poison is another man's meat. Using azithromycin-induced phospholipidosis to promote ocular surface health. Toxicology. 2014;320:1-5.

17. Igami TZ, Holzchuh R, Osaki TH, Santo RM, Kara-Jose N, Hida RY. Oral azithromycin for treatment of posterior blepharitis. Cornea. 2011;30(Oct):1145-9.

18. Foulks GN, Borchman D, Yappert M, Sung-Hye K, McKay JW. Topical azithromycin therapy of meibomian gland dysfunction: clinical response and lipid alterations. Cornea 2010;29:781.

19. Ciloglu E, Özcan AA, Incekalan T, Unal F. The role of topical azithromycin in the treatment of meibomian gland dysfunction. Cornea. 2020;39(Mar):321-4.
20. Macsai MS. The role of omega-3 dietary supplementation in blepharitis and meibomian gland dysfunction (an AOS thesis). Trans Am Ophthalmological Soc. 2008;106:336.

21. Molina-Leyva I, Molina-Leyva A, Bueno-Cavanillas A. Efficacy of nutritional supplementation with omega-3 and omega- 6 fatty acids in dry eye syndrome: a systematic review of randomized clinical trials. Acta Ophthalmol. 2017;95:e677-85.

22. Oydanich M, Maguire MG, Pistilli M, Hamrah P, Greiner JV, Lin MC, et al. Management study research group. effects of omega-3 supplementation on exploratory outcomes in the dry eye assessment and management study. Ophthalmology. 2020;127:136-8.

23. Rhee MK, Mah FS. Clinical utility of cyclosporine (CsA) ophthalmic emulsion $0.05 \%$ for symptomatic relief in people with chronic dry eye: a review of the literature. Clin Ophthalmol (Auckl, NZ). 2017;11:1157.

24. Kim HY, Lee JE, Oh HN, Song JW, Han SY, Lee JS. Clinical efficacy of combined topical $0.05 \%$ cyclosporine a and $0.1 \%$ sodium hyaluronate in the dry eyes with meibomian gland dysfunction. Int J Ophthalmol. 2018;11:593.

25. Semba CP, Gadek TR. Development of lifitegrast: a novel T-cell inhibitor for the treatment of dry eye disease. Clin Ophthalmol (Auckl, NZ). 2016;10:1083.

26. Liu J, Sheha H, Tseng SC. Pathogenic role of demodex mites in blepharitis. Curr Opin Allergy Clin Immunol. 2010;10(Oct):505.

27. Fromstein SR, Harthan JS, Patel J, Opitz DL. Demodex blepharitis: clinical perspectives. Clin Optom. 2018;10:57.

28. Arita R, Suehiro J, Haraguchi T, Maeda S, Maeda K, Tokoro H, et al. Topical diquafosol for patients with obstructive meibomian gland dysfunction. Br J Ophthalmol. 2013;97:725-9.

29. Albietz JM, Schmid KL. Randomised controlled trial of topical antibacterial Manuka (Leptospermum species) honey for evaporative dry eye due to meibomian gland dysfunction. Clin Exp Optom. 2017;100:603-15. 